\title{
Alter
}

Revue de phénoménologie

$27 \mid 2019$

Patočka

\section{L'ambiguïté du monde}

\section{Renaud Barbaras}

\section{(2) OpenEdition}

Journals

Édition électronique

URL : https://journals.openedition.org/alter/1858

DOI : $10.4000 /$ alter.1858

ISSN : 2558-7927

\section{Éditeur :}

Association ALTER, Archives Husserl (CNRS-UMR 8547)

\section{Édition imprimée}

Date de publication : 1 novembre 2019

Pagination : 13-28

ISBN : 978-2-9550449-5-7

ISSN : 1249-8947

\section{Référence électronique}

Renaud Barbaras, «L'ambiguïté du monde », Alter [En ligne], 27 | 2019, mis en ligne le 22 décembre 2020, consulté le 14 juin 2021. URL : http://journals.openedition.org/alter/1858 ; DOI : https://doi.org/ 10.4000/alter. 1858

Ce document a été généré automatiquement le 13 juin 2021

Revue Alter 


\title{
L'ambiguïté du monde
}

\author{
Renaud Barbaras
}

1 Mon interrogation portera sur le statut et la place de la perspective cosmologique chez Patočka, plus précisément sur son aptitude à rendre compte de la phénoménalité dans la plénitude de son sens, à savoir à la fois comme phénoménalité anonyme du monde et ce que je nommerai phénoménalité secondaire, qui renvoie à l'apparaitre à un sujet, ou encore à l'apparaître comme tel. Ce qui est en question n'est donc autre que la possibilité de penser la subjectivation dans un cadre strictement cosmologique. Or, je voudrais montrer que, en dépit de certaines formulations ambiguës, Patočka échoue à rendre compte de l'apparaître subjectif dans le cadre cosmologique et que sa phénoménologie, en son stade d'achèvement maximal, demeure traversée par une dualité entre l'apparaître anonyme et la compréhension, dualité qui renvoie en dernière instance au fait que l'approche cosmologique de provenance finkéenne s'intègre dans une perspective d'ensemble qui demeure heideggerienne, comme Patočka le reconnaît d'ailleurs au moins une fois ${ }^{1}$.

2 Il est incontestable que Patočka est largement influencé par la perspective finkéenne, qui vise à substituer à la différence ontologique une différence cosmologique, c'est-àdire à aborder l'étant depuis son appartenance constitutive au monde, monde dont le sens est lui-même déterminé par la radicalité de cette approche. Il s'agit d'une totalité, d'un omni-englobant dans la mesure où tout étant lui appartient mais qui, pour cette raison même, ne saurait être conçu ni comme une totalité additive, ni comme un grand objet. Le monde n'est monde, c'est-à-dire ne contient tout, que dans la mesure où il n'est pas autre que ce qu'il contient, sa différence avec tout étant exclut toute altérité, sans quoi il serait lui-même un étant. Dès lors, le monde n'est autre que ce qui unifie les étants en leur multiplicité même, l'unité du multiple si l'on veut mais une unité absolument immanente au multiple, qui ne conjoint qu'en disjoignant. Il est donc entre les étants à la fois au sens où il les unit comme une étoffe et les sépare comme une frontière: il est comme un tissu qui est sa propre déchirure, bref l'Ajointement universel. Par là-même, il doit être conçu comme un élément plutôt que comme une totalité, ou plutôt une totalité qui est un élément. En effet, le monde ne peut tout contenir que dans la mesure où il est ce dont tous les étants sont faits, leur texture commune, texture qui accepte ou plutôt permet l'infinie diversité des étants. Il est donc 
une forme universelle qui est en même temps la matière ultime, matière de tout ce qui peut être, par conséquent l'indistinction absolue de la matière et de la forme. Cependant et enfin, cette absolue immanence du monde aux étants, immanence qui n'en compromet pas l'unité, ne peut être pensée que dynamiquement. Si le monde n'est pas autre que chaque étant sans se confondre avec eux, c'est seulement dans la mesure où il n'est que la puissance qui les produit et qui, les produisant, se produit en eux. Dès lors, l'excès à la fois inassignable et irréductible du monde sur les étants mondains renvoie à l'excès de la puissance comme surpuissance ou réserve absolue sur tout ce qu'elle produit : la puissance n'est autre que ses œuvres mais elle n'est pas ses œuvres puisqu'elle les produit. Or, dans la mesure où les étants procèdent tous de cette puissance une, où elle se produit comme différenciation des étants, il faut conclure que le monde n'est rien d'autre, en dernière analyse, que cet incessant procès d'individuation des étants ou, plus précisément, ce qui s'individue en individuant les étants (en se différenciant). Comme l'écrit Fink, que je ne cite que parce que la proximité avec Patočka est quasi-littérale: «La vie du monde dispose de tout étant individuel ; elle fait naître et passer, croître et disparaître, elle met les choses dans leur être individualisé et les en enlève à nouveau - elle se produit comme individualisation universelle de tout étant $»^{2}$.

3 Totalité, ajointement, élémentarité et puissance individuante, tels sont les traits sous lesquels peut être pensé le monde, étant entendu qu'il se donne phénoménologiquement d'abord comme profondeur interne, être là de tout étant : les traits que je viens d'énoncer reviennent donc à développer cette présence originaire dans les termes d'une appartenance originaire. Dès lors, dans la mesure où nous nous situons dans une perspective phénoménologique, où il s'agit donc de décrire l'apparaître de tout étant en tant que cet apparaître enveloppe celui du monde, force est de reconnaître que le procès d'individuation par lequel nous avons caractérisé en dernière analyse le monde est ipso facto un procès de phénoménalisation. Autrement dit, le monde paraît au sein des étants tout en demeurant voilé en eux, ou encore s'efface derrière eux dans la mesure où, en les différenciant, c'est-à-dire en les individuant, il se produit lui-même. Il s'agit donc de comprendre en quoi ce procès d'individuation est bien un procès de phénoménalisation. Or l'apparaître peut être défini comme une sortie de l'occultation et de la manière dont on pense celle-ci dépend dès lors celle dont on comprend celui-là. Si l'occultation est comprise comme recouvrement, par une couche intermédiaire ou par le sujet lui-même, l'apparaître ne peut être pensé que comme dévoilement, supposant l'action d'un sujet, que celui-ci soit conscience ou Dasein. Mais il y a une autre manière, plus radicale et plus secrète, d'être occulté : non plus par recouvrement, interposition d'une couche étrangère mais par confusion avec l'entourage, absence de délimitation, indistinction. L'étant est alors caché non pas parce que quelque chose le dissimule mais parce qu'il n'est pas encore défini, détaché de son entourage, circonscrit, même s'il est déjà là tout entier, au moins virtuellement. L'apparaitre, comme sortie de l'occultation, est alors synonyme de dépassement de l'indistinction, c'est-à-dire de délimitation ou de circonscription. Or, cette délimitation ne saurait être rien d'autre qu'une différenciation et donc une individuation qui, comme telle, peut et même doit être l'œuvre de la puissance anonyme du monde et non plus de la découverte par un sujet. Dire de la chose qu'elle paraît c'est dire qu'elle paraît elle-même (et non pas que disparaît ce qui l'empêchait d'être visible) et dire qu'elle paraît elle même, c'est tout simplement signifier qu'elle ne se confond plus avec son entourage, qu'elle se détache de l'élément anonyme au sein 
duquel elle est immergée, bref qu'elle s'individue. Il devient alors compréhensible que la puissance individuante du monde soit ipso facto une puissance phénoménalisante, de telle sorte que les conditions de l'étantité de l'étant sont en même temps celles de son apparaître. Il faut donc reconnaître un plan de phénoménalité anonyme, celui d'un apparaître qui n'est encore apparaître à personne pour autant qu'il se confond avec le procès d'individuation de l'étant par le monde. Or, il est incontestable que Patočka reprend pleinement à son compte cette version cosmologique de la phénoménalité, au point qu'il l'explicite mieux que Fink ne le fait lui-même. Ainsi, il écrit, en résumant la conception finkéenne du monde comme «cadre préalable, total, non-individué, de toute individuation » que :

La manifestation des choses, rendue possible par ce cadre, n'est pas manifestation pour le sujet, mais bien manifestation comme entrée dans la singularité, devenir. C'est une manifestation dans laquelle les choses singularisées sont elles-mêmes intérieurement indifférentes, une manifestation qui n'est pas manifeste à ellemême, une manifestation plongée dans l'obscurité nocturne de l'étant et de l'être originaire $^{3}$.

Toute la question se concentre dès lors sur la place de l'étant singulier que nous sommes. Il est incontestable qu'il appartient au monde, au même titre que les autres étants et que, dans cette mesure, il relève du procès mondain d'individuation, ce que Fink reconnaît clairement: "L'homme appartient lui aussi à ce processus; incontestablement, il est une chose finie; il est même la chose "la plus finie" " ${ }^{4}$. Mais le rapport de cet étant au monde n'est pas seulement d'appartenance au sens de l'intramondanéité, de la simple insertion dans le monde ; il se rapporte également activement à lui au sens où il le fait paraître, où il saisit le monde comme monde, rapport que Fink, et Patočka à sa suite, vont définir comme compréhension. Il s'agit alors de savoir dans quelle mesure cette dimension, qui caractérise en propre le sujet que nous sommes, peut être comprise à partir du monde tel qu'il a été défini, peut être inscrite dans le procès d'individuation. La compréhension du monde, pour parler comme nos auteurs, peut-elle être une production de la surpuissance du monde? Notons au passage que le traitement de cette question est indissociable de la manière dont le rapport du sujet au monde est caractérisé : il n'est pas certain que le concept de compréhension soit compatible avec l'insertion de l'homme dans la monde et, à l'inverse, la question est bien de savoir quel est le mode d'être propre d'un sujet qui, se rapportant au monde, n'échappe pourtant d'aucune façon à son emprise. Quoi qu'il en soit, l'enjeu n'est autre ici que celui de la pertinence, c'est-à-dire de la puissance explicative de la perspective cosmologique. Si le monde nomme bien le sens d'être ultime de tout étant et s'il est vrai que nous appartenons au monde, même si c'est de manière singulière, alors il doit être possible de rendre compte de notre mode d'être à partir de celui du monde, au titre de production de celui-ci. Si, à l'inverse, notre mode d'être ne peut être reconduit à celui du monde, force est alors de reconnaître une instance irréductible au monde et de conclure à l'échec de la cosmologie, échec qui signifie nécessairement un retour en force, même si c'est sous une forme déplacée, du dualisme métaphysique. On peut dire que l'œuvre de Patočka, à la suite de celle de Fink, est traversée par cette interrogation, soucieuse qu'elle est d'en finir avec toute instance subjective autonome, mais force est de constater aussi que Patočka ne parvient pas à surmonter le dualisme que je viens d'évoquer, dualisme qui est, en dernière analyse, celui de la cosmologie et de l'ontologie fondamentale. 
5 Patočka commence par affirmer, à la suite de Fink et ce à maintes reprises, que « Notre propre individuation appartiendrait elle aussi à cet univers de l'individuation primordiale englobant tout ce qui se dévoile initialement dans sa simple advenue, dans son déploiement, sa naissance et sa disparition, sans participation intérieure à l'être, sans intérêt pour l'être $»^{5}$, ce qui revient à dire que, comme toutes les autres choses, l'étant que nous sommes émerge au sein du monde puis disparaît, commence et finit. Aux yeux de Patočka, cette appartenance à l'univers de l'individuation primordiale renvoie à notre vie biologique, à notre organisme en tant qu'il naît et meurt et il parlera ailleurs d'individuation physique à propos de la séparation d'avec le corps de la mère ${ }^{6}$, par différence avec l'individuation "psychique » qui concerne l'émergence du sujet en son autonomie. Notons que les choses sont ici déjà jouées : verser l'identité biologique au compte de l'individuation primordiale, c'est-à-dire mondaine, c'est évidemment dessiner la place d'une individuation qui y échappe, pour autant que notre existence ne se confond pas avec notre identité organique et c'est donc entériner la dualité en nous entre ce qui, strictement corporel, appartient à l'individuation mondaine et ce qui y échappe. C'est ce qui apparaît clairement à la suite du fragment que nous venons de citer :

La caractéristique du mouvement qui nous est spécifique serait cependant la nonindifférence à l'être, l'intérêt pour l'être propre et, conjointement, pour l'être de l'étant en général, sur le fondement d'une manière nouvelle dont l'être conditionne l'étant - non plus simplement dans son émergence et sa disparition, mais bien en tant que clarté rendant possible la rencontre en dedans, à l'intérieur de l'univers.

On voit surgir ici la détermination heideggerienne du Dasein dans sa double dimension (compréhension de son propre être/compréhension de l'être) qui vient en quelque sorte se greffer sur l'existence biologique, dessinant ainsi une dualité au sein du mouvement : il y a le mouvement de la vie, mouvement d'émergence et de disparition, qui procède directement du mouvement cosmique et il y a ce mouvement singulier qu'est l'existence comme pouvoir être et compréhension. Si le premier signe l'alliance de la biologie et de la cosmologie, le second échappe à celle-ci même si, comme nous le verrons, Patočka tentera de penser leur unité.

7 Ainsi, l'étant que nous sommes a ceci de propre qu'il s'individue lui-même en se mettant à part du procès mondain, cette mise à part n'étant que l'autre face d'un rapport au tout du monde, à la totalité comme telle. En vérité, c'est bien du même sens de l'individuation comme limitation ou délimitation dont il s'agit de part et d'autre : tout comme les étants intra-mondains sont délimités par le procès du monde, le monde lui-même se trouve délimité, c'est-à-dire totalisé, par le surgissement de l'étant que nous sommes, dans la mesure où l'existence de celui-ci peut être caractérisée comme rapport à la totalité, et cela sous une forme qui est celle de la Befindlichkeit avant d'être celle de la compréhension. Cependant, si la première limitation est l'œuvre du monde, la seconde ne peut l'être puisqu'elle porte sur le monde et que celui-ci ne peut se totaliser lui-même à partir de lui-même, sans quoi il faudrait qu'il sorte de lui-même et cesse par là-même d'être un omni-englobant. Pour autant, cette totalisation ne renvoie pas à l'activité constituante ou totalisante d'un sujet; la limitation de la cosmologie, inhérente à celle du monde comme tel, n'est pas au service du subjectivisme mais de sa négation. Telle est la continuité négative qui se manifeste entre les deux plans d'individuation : «Si l'on est en droit de poser une partie de l'apparition contingente du monde, de la 'limitation' du tout du monde, comme indépendante à l'égard du sujet, la même chose vaut aussi pour la limitation qui englobe le monde entier ${ }^{8}$. En effet, 
comme il apparaît clairement dans le texte dont nous sommes partis, la singularité de notre rapport au monde, qui est "intérêt " pour l'être et compréhension du tout du monde (dimensions qu'il faudrait articuler), a pour fondement la «manière nouvelle dont l'être conditionne l'étant ». Autrement dit, la singularité de notre mouvement, conduisant à une mise à part du monde par totalisation, plonge dans l'être lui-même ; c'est l'être qui se manifeste et, pour ainsi dire, se produit dans notre singularité d'existants, c'est sa différence qui se fait jour dans notre individuation, et celle-ci n'en est en vérité que l'envers. Ainsi, notre individuation échappe au procès du monde non pas parce qu'elle renverrait à une instance hors monde, telle une subjectivité transcendantale mais, au contraire, parce qu'elle s'enracine plus profondément que le monde, dans un procès d'auto-manifestation de l'être, dans un mouvement qui n'est plus cosmologique mais ontologique. Alors que le monde se manifeste en se produisant sous la forme des étants intra-mondains, y compris des vivants que nous sommes aussi, l'être se manifeste à travers les étants que nous sommes exclusivement. Autrement dit et plus précisément, il y a "deux manières fondamentales dont l'être découvre l'étant $»^{9}$ qui donnent lieu à deux types d'étants distincts : d'une part, en les produisant sous la forme d'étants intramondains, d'autre part, en les éclairant en rendant possible la rencontre, autrement dit en faisant être le Dasein. Au premier niveau, l'être se produit comme monde, c'est-à-dire différenciation d'étants indifférents au monde ; au second niveau, l'être paraît en donnant lieu à un étant non-indifférent au monde et par conséquent capable de le totaliser. Soulignons au passage que ces deux procès correspondent aux deux modalités de désoccultation que nous avons distinguées : alors que les étants intra-mondains paraissent en tant qu'ils sont individués par le monde, l'être et le monde comme tel paraissent en tant qu'ils sont dévoilés par le Dasein.

Quoi qu'il en soit, on le voit, non seulement le sujet que nous sommes relève de l'ontologie alors que le vivant, à l'instar des autres étants, relève de la cosmologie mais on assiste à une subordination qui prend la forme d'une intégration de la cosmologie à l'ontologie, intégration qui est incontestablement propre à la phénoménologie de Patočka. Le monde se produit sous la forme des étants mais il y a ce qu'il nomme parfois un "fond du monde", qui n'est autre que l'être et qui se dévoile par la médiation du Dasein. De part et d'autre, nous avons affaire à deux procès de nature différente et donc à deux modes d'apparaître radicalement distincts : on pourrait dire que tout l'effort de Patočka a consisté à tenter de penser l'unité de ces deux dimensions, bref à rendre compatibles Fink et Heidegger. Il y a bien un seul sens de l'individuation, comme délimitation, mais bien deux sens de la manifestation: l'apparaître primaire est l'œuvre du monde, l'apparaître secondaire est l'œuvre de l'être par l'entremise du Dasein. Il suit finalement de là que le monde est subordonné à l'être, est pour ainsi dire un moment de l'être et c'est finalement sur ce seul point que Patočka se sépare de Heidegger, là encore à la suite de Fink, refusant de reconduire le monde au seul être-au-monde du Dasein. Patočka formule la situation au moins une fois, dans un texte d'une étonnante lucidité :

Voilement de l'être - en ce sens qu'il passe inaperçu, qu'il est dévoilement même, mais aussi qu'il ne met jamais en œuvre toutes ses possibilités, ne montrant que des possibilités de phénoménalisation. L'être est donc $1 /$ le tout préalable de l'espacetemps qui, compte tenu de son caractère préalable et non composé, toujours déjà présupposé par toutes les singularités, ne peut être réduit aux choses étantes qu'il rend possible ; 2/ le tout préalable de la compréhension qui rend possible l'être-àdécouvert tant de l'univers en totalité que des singularités, tout qui appartient au premier comme son aspect de sortie hors de soi, de dévoilement de soi ${ }^{10}$. 
En d'autres termes, le voilement de l'être renvoie à la fois à la réserve de puissance du monde et à son occultation en tant qu'être, étant entendu que la compréhension désigne le mouvement par lequel le monde tout entier sort de lui-même pour se dévoiler. En ce sens, on pourrait dire que l'être n'est rien d'autre que le monde en tant qu'il est capable de sortir de soi pour se comprendre. Mais on voit que cette dimension échappe à la caractérisation strictement cosmologique du monde et que le mouvement singulier auquel elle donne lieu et sur lequel elle repose, à savoir le mouvement de l'existence, échappe totalement au procès mondain.

10 Bien entendu, toute la question est de savoir jusqu'à quel point il est possible de concilier ces deux dimensions et donc ces deux sens de l'apparaître. Le procès d'individuation des étants n'a rien à voir avec celui de l'auto-manifestation de l'être et on se demande comment ces deux procès peuvent être le même ou avoir la même source, comment ils peuvent coïncider dans l'être ou comme être. On ne peut s'empêcher de penser que Patočka demeure prisonnier d'une forme résiduelle de subjectivisme, en dépit bien sûr de toutes ses affirmations, dans la mesure où il ne parvient pas à verser l'apparition du monde au compte du monde, où il la fait au contraire reposer sur un étant qui n'a pas grand chose à voir avec les autres étants - et le fait que cette apparition s'enracine ultimement dans l'être et que le Dasein qui la médiatise ne soit pas une conscience n'y change pas grand chose. En vérité, une phénoménologie authentiquement a-subjective est une phénoménologie qui parviendrait à en finir avec cette situation d'exception, bref qui parviendrait à rendre compte du sujet, en tant qu'opérateur de l'apparition proprement dite, à partir du procès même du monde au lieu de subordonner subrepticement celui-ci à l'être afin de rendre compte de son apparaître à... Bref, on le voit ici, par-delà leur opposition apparente, il y a une complicité secrète entre le subjectivisme et l'ontologie fondamentale, qui repose sur leur incapacité à rendre compte de la phénoménalisation du monde comme tel, ou encore de la subjectivation à partir de ce seul monde. Evidemment, un tel programme s'expose sans cesse au risque du naturalisme et toute la difficulté, que ni les uns ni les autres n'ont osé affronter, est de savoir comment on peut y échapper. Soulignons enfin, sans pouvoir nous y attarder, que c'est cette articulation singulière de l'être et du monde qui permet de comprendre les affirmations tardives de Patočka sur le rapport entre métaphysique et phénoménologie, entre l'être et le phénomène. Selon lui, le surgissement de la subjectivité et donc de la phénoménalité proprement dite n'est inscrit d'aucune façon, pas même sur le mode téléologique, dans la réalité, ce qui revient à dire qu'un monde sans l'homme est parfaitement concevable. Néanmoins, il y a une forme de co-détermination de l'être par la phénoménalité qui fait que l'être sans phénomène apparaît comme manquant de quelque chose, comme en défaut. Ainsi, écrit Patočka :

[...] se trouve ancré dans le fond même du monde, un étant qui n'est pas nécessaire, mais qui fait nécessairement que le nécessaire émergeant devant lui apparait comme un mode d'être déficient, quelque chose qui, certes, apparaît et qui est, mais auquel manque la clarté de l'intéressement à soi-même, le se-rapporter intérieurement à soi et à l'autre ${ }^{11}$.

11 Une telle affirmation ne peut être comprise qu'à la lumière de l'ambiguïté constitutive du monde que nous avons mise en évidence et qui se résume à ceci que le monde est à la fois monde et être. En effet, du point de vue du monde, qui est le règne de la nécessité, le surgissement du sujet est évidemment contingent : rien dans l'essence du monde comme tel n'appelle la subjectivation. Il n'en reste pas moins qu'il y a un en- 
deçà du monde qui appelle un au-delà du monde, autrement dit que le fond de monde ou le monde comme être appelle son propre dévoilement par l'entremise d'un étant qui est intéressé à lui-même. Telle est la raison pour laquelle ce qui appartient simplement au monde apparaît comme en défaut, l'être comme tel appelant une phénoménalité qu'il ne peut produire comme monde. En vérité, la première partie de la thèse, à savoir la contingence du sujet par rapport au procès du monde est incontestable; ce qui fait problème est plutôt la volonté de reconduire ce sujet à une auto-manifestation de l'être. En effet, une démarche strictement cosmologique ne devrait-elle pas s'en tenir au contraire à la contingence de ce surgissement par rapport au procès du monde et en tirer toutes les conséquences, quitte à creuser l'écart entre ce surgissement comme tel et le mouvement du monde au lieu de tenter de les réconcilier dans l'être?

Comme nous l'avons déjà aperçu, le mouvement est «le moyen terme entre les deux manières fondamentales dont l'être découvre l'étant $\aleph^{12}$, à savoir en le produisant l'être a alors pour nom le monde - et en le faisant paraître. En effet, le procès mondain d'individuation s'accomplit sous la forme du mouvement ontogénétique par lequel un étant naît puis disparaît, mais aussi et par là même sous celle de l'ensemble des mouvements qui le traversent et en lesquels consiste son existence. Autrement dit, c'est nécessairement sous les espèces d'un mouvement effectif que s'atteste l'appartenance d'un étant quelconque au monde (le repos n'étant donc toujours qu'une modalité du mouvement, y compris pour les étants non-vivants, ce repos consistant alors en ce qui est impliqué par leur inertie). Ceci est a fortiori vrai pour l'étant que nous sommes dans la mesure où l'être en vie se manifeste d'emblée par un ensemble de mouvements. La question de l'articulation entre l'apparaître anonyme du monde et son apparaître proprement dit se concentre alors dans la question du passage du mouvement du vivant que nous sommes à ce mouvement singulier, dont Patočka emprunte la caractérisation au pouvoir-être heideggerien et qui se déploie comme compréhension du monde. Comment passer de ces mouvements effectifs que sont les premiers mouvements du vivant nouvellement né, mouvements qui, comme tels, ne semblent encore rien dévoiler, au mouvement de dévoilement de l'étant? Comment passer de ces mouvements que Patočka qualifie parfois d'instinctifs au mouvement que l'on pourrait qualifier d'intentionnel et qui, selon Patočka, engage un rapport au tout du monde?

Qu'un tel passage soit pensable suppose d'abord que, de part et d'autre, le sens du mouvement soit univoque, qu'il y ait un sens originaire du mouvement qui vaille à la fois et également pour le mouvement vivant ou instinctif et pour la compréhension. Or, il serait facile de montrer que Patočka ne parvient pas à penser un tel sens, que l'unité entre ce qu'il nomme mouvement de l'existence et le mouvement comme action effective dans le monde demeure analogique ou métaphorique. En d'autres termes, Patočka maintient une différence entre les mouvements proprement dits, mouvements du corps qui ont lieu dans le monde, d'une part, et les mouvements de l'existence, d'autre part, qui, quant à eux, impliquent une relation, même implicite, au tout du monde. Cette différence n'est autre que celle qui passe entre un processus intramondain qui continue à avoir la puissance mondifiante pour source et un mouvement phénoménalisant, nécessairement en rupture avec le monde puisque, on l'a vu, s'enracinant dans le procès par lequel l'être sort de lui-même. Il y a donc une nécessaire dualité des mouvements, car on ne voit pas comment l'ambiguïté constitutive du monde pourrait être surmontée au plan des mouvements auxquels elle 
donne lieu. Plus encore et de manière révélatrice, Patočka en vient à établir une hiérarchie de type téléologique entre les mouvements concrets et les mouvements existentiels :

Chaque étant est pratique, actif, chacun est impliqué dans des complexes de relations 'causales', chaque réalité est un acte effectif, une manifestation. Mais seule est active au sens éminent la réalité qui n'est pas indifférente à son propre égard, celle qui ne peut se réaliser que dans la non-indifférence au fait qu'elle est et qu'elle est comme elle est - celle qui peut avoir une compréhension pour son être propre (ce qui signifie, conjointement, pour l'être en général, pour l'être en totalité, pour le monde) ${ }^{13}$.

14 Ainsi, même si d'autres étants que nous sont actifs, le sens éminent de l'activité consiste dans la compréhension.

On assiste ici à une sorte d'inversion par laquelle le sens éminent du mouvement est référé à l'activité de dévoilement, de telle sorte que ce qui est simplement en mouvement et relève donc du sens originaire du mouvement doit finalement être considéré comme n'étant pas pleinement actif et donc pas pleinement en mouvement. L'essence du mouvement est bien du côté de la compréhension et non de l'action effective, de cela dont la source est l'être et non le monde. Telle est la raison pour laquelle Patočka peut parler de mouvement de l'existence en un sens qui n'est aucunement métaphorique: à ses yeux, le mouvement est d'abord l'existence ellemême comme compréhension et c'est alors ce que nous entendons couramment par mouvement qui doit être compris en un sens métaphorique, ou plutôt métonymique puisqu'il se contente de sous-tendre les mouvements existentiels au titre de leur partie matérielle ou de leur condition. En d'autres termes, si Patočka insiste en effet sur la corporéité motrice comme existential du Dasein, c'est au titre de condition de possibilité et de moment interne de ce qui demeure finalement l'existential essentiel, à savoir la compréhension, qui est médiatisée par la Befindlichkeit, à laquelle renvoie finalement le premier mouvement de l'existence ${ }^{14}$. Le mouvement concret, corporel, est donc seulement le tribut qu'il faut payer à la cosmologie ; il est le point d'articulation entre les deux modalités d'ouverture ou de conditionnement de l'étant, c'est-à-dire la manière dont le mouvement de compréhension, seul mouvement véritable, s'inscrit dans le monde et s'insère ainsi dans le procès d'individuation du monde. Mais, on le voit, une dualité insurmontable persiste au plan du mouvement; il demeure évidemment impossible d'articuler au niveau du sujet individuel et de son mouvement ce qui ne l'a pas été au plan des deux procès de manifestation. Autrement dit, Patočka leste en quelque sorte le Dasein avec un corps moteur qui l'inscrit dans le monde et le rend tributaire de ses processus, mais il ne parvient d'aucune façon à rendre compte de la phénoménalité secondaire à partir de ce mouvement vivant. Un abîme demeure entre ce par quoi nous appartenons au monde, notre vie proprement dite, et ce par quoi nous le phénoménalisons, notre existence. Ici encore, il est difficile de nier que Patočka conserve, sous une forme certes déplacée sur le plan moteur, la dualité métaphysique du corps et de l'esprit, dualité qu'il reconnaît sous les termes d'individuation physique (par le monde) et d'individuation psychique (par l'existence).

C'est évidemment au niveau de ce qu'il nomme le premier mouvement de l'existence que Patočka tente de penser, de manière cette fois génétique, la continuité entre le mouvement vivant et le mouvement existentiel, le passage de l'individuation dans et par le monde à l'individuation comme intérêt pour soi et pour le monde. C'est aussi à ce niveau que l'échec est le plus patent. Comme on le sait, ce mouvement prend son point 
de départ dans ce que Patočka nomme l'incomplétude de l'individuation physique, par quoi il entend l'état d'impuissance dans lequel se trouve le nouveau-né humain, incapable qu'il est de subvenir à ses besoins. C'est pourquoi la condition de l'existence de ce sujet est l'accueil par une communauté, qui est d'abord celle de la famille. C'est à la faveur de cette insertion dans une relation intersubjective que les mouvements d'abord instinctifs (c'est le terme de Patočka) se muent en mouvements signifiants, que le corps vivant accède au rang de corps vécu. Ce premier mouvement est «impulsion vers l'attachement » et implique comme tel une "consonance avec l'aspect global du monde $»^{15}$; il rend possible un premier rapport à la totalité comme telle sur le mode affectif, " il est mouvement de la disposition, du se trouver ${ }^{16}$. Ainsi, Patočka procède à une sorte de genèse de la Befindlichkeit heidegerienne en faisant surgir la dimension de l'affectivité à la faveur de l'accueil d'un être impuissant par ses proches; il tente d'enraciner les existentiaux heideggeriens dans ce qui est à ses yeux le premier existential, à savoir la corporéité motrice. Toute l'analyse du premier mouvement vise donc à saisir en quelque sorte sur le vif le passage des mouvements proprements physiques, provenant de l'individuation primordiale, à des mouvements signifiants et susceptibles de comprendre la totalité, ou encore le passage du mouvement comme effet au mouvement comme dévoilement. Ainsi :

Le 'je fais' présuppose un 'je peux' et se déploie sur sa base. Je fais toujours ce que je peux, et le miracle du faire tient à ce qu'il excède ce 'je peux' originaire. Si je ne peux d'abord qu'attirer par mes cris l'attention des autres, la réponse à cet appel en fait une découverte de la nourriture et une manière de supprimer le déplaisir et l'inconfort; les mouvements involontaires des membres deviennent un éloignement et un rapprochement, un maniement des choses ${ }^{17}$.

Cette analyse du premier mouvement, où se joue le passage de l'individuation mondaine à la compréhension du monde et, partant, l'articulation de la cosmologie et de l'ontologie, soulève de nombreuses difficultés. Tout d'abord, en référant cette métamorphose des mouvements involontaires en mouvements signifiants à l'accueil par l'autre, on repousse le problème plutôt qu'on ne le résout : comme chez Platon, on se demande qui a bien pu accueillir le premier autre. D'autre part, comme Patočka le note discrètement, cet accueil par les autres suppose une acceptation de la part de celui qui est accueilli; si cette inscription dans l'intersubjectivité conditionne l'autonomie, elle la suppose tout autant. Enfin et surtout, le passage d'un type de mouvement à l'autre tel que le décrit Patočka est tout simplement impensable. Dans la mesure où il s'agit pour lui de rendre compte de l'émergence de la compréhension à partir du simple organisme vivant, ou encore du rapport au monde à partir de l'insertion dans le monde, il tente de rapprocher au maximum les mouvements vivants des mouvements mondains en les pensant comme mouvements instinctifs afin de faire reposer exclusivement sur l'acceptation leur mutation en mouvements existentiels ou humains. Mais cette tentative est vouée à l'échec; si l'acceptation permet certes une transformation, celle-ci demeurerait néanmoins impossible si les mouvements vivants n'enveloppaient pas déjà un rapport au monde, en quoi ils se distinguent radicalement des mouvements non-vivants. En d'autres termes, c'est évidemment l'usage du concept d'instinct qui pose ici problème, tout au moins en tant qu'il vise à signifier un mouvement quasi-mécanique, aussi proche que possible des mouvements mondains car, en vérité, pas plus chez l'homme que chez l'animal, le mouvement vivant ne peut se réduire à ce mouvement aveugle. C'est donc de deux choses l'une : ou bien la vie du nouveau-né s'épuise dans ces mouvements aveugles et n'enveloppe aucun rapport au monde et aux autres, mais alors aucun accueil n'y changera jamais quoi que ce soit; ou 
bien cet accueil rend possible cette marche vers l'autonomie que décrit Patočka, mais c'est dans la mesure exacte où le vivant humain était déjà en rapport avec le monde, où ses mouvements étaient déjà abyssalement différents des mouvements intramondains. Bref, le vivant ne peut se muer en existant que si sa vie se distingue de celle du monde, autrement dit est déjà existence. L'erreur ici est de vouloir engendrer l'existence à partir de la vie, d'une vie saisie en continuité avec le procès mondain ; elle est de ne pas voir que, avec la vie du vivant le plus simple, on a déjà quitté le plan du mouvement mondain, que le moindre mouvement vivant est radicalement distinct des mouvements intra-mondains, que le se-mouvoir, aussi sommaire et aveugle soit-il, est profondément différent de l'être-mu et contient en germe l'ouverture au monde que Patočka veut réserver au seul existant humain. Autrement dit, en dépit de son souci de faire une genèse de l'ouverture au monde à partir de l'insertion dans le monde et donc de construire un concept unitaire du mouvement intégrant ces deux rapports au monde, il apparaît clairement que la différence pertinente aux yeux de Patočka passe entre se mouvoir dans le monde et comprendre le monde, entre vie et existence, différence qui apparaît alors comme l'ultime avatar, déplacé au plan du mouvement, des dualismes métaphysiques classiques.

Il apparaît donc clairement que, en dépit de ses tentatives de "cosmologiser " l'existence humaine en recourant à un concept de mouvement censé enraciner l'existence dans le monde, Patočka s'avère incapable de rendre compte de la totalité des dimensions de l'existence dans un cadre cosmologique. Autant dire que, en dépit de sa tentative de les unifier, la coupure entre la cosmologie et l'ontologie demeure insurmontable, qu'il ne devient alors possible de faire droit à notre existence que par une intégration de la cosmologie à l'ontologie fondamentale. On voit a contrario que la condition fondamentale d'une approche exclusivement cosmologique est la reconnaissance de l'indivisibilité véritable du mouvement vivant, c'est-à-dire de l'indistinction de la vie et de l'existence. Cela signifie d'abord qu'il faut renoncer au comprendre pour caractériser l'existence humaine, à cette projection de l'herméneutique dans le Dasein qui conduit à le scinder inexorablement de la vie. Comme les autres vivants, l'homme est caractérisé par un pouvoir de phénoménalisation qui est corrélatif de son auto-mouvement. Il n'est plus agi par le monde mais agit dans le monde et dans cette mesure le fait paraître, ou plutôt le mouvement vivant est indissociablement avancée et dévoilement: c'est cette indistinction que je recueille dans le concept de désir. Cependant, la coupure au sein du mouvement n'est pas abolie mais déplacée : elle ne passe plus entre l'instinct et la compréhension mais entre les mouvements intra-mondains et les mouvements vivants, entre l'être-mû de l'étant non-vivant et le se-mouvoir du vivant. L'objet propre et pour ainsi dire le défi d'une cosmologie est alors de penser cette émergence ou ce passage à partir du procès même du monde, ou plutôt, puisque nos mouvements ne sont pas strictement engendrables à partir de ceux du monde sauf à tomber dans le naturalisme, de penser cette émergence en n'introduisant rien d'autre que le monde lui-même. Or, tel est le point fondamental, alors que le passage du se-mouvoir à la compréhension signifiait un gain et non une perte, de telle sorte qu'il y a plus dans la compréhension que dans le simple mouvement, le passage des mouvements mondains à l'automouvement vivant s'effectuera sur le mode négatif de la privation ou de la limitation, et c'est pourquoi la transition de la cosmologie à la biologie, qui est ce qui importe à nos yeux, ne fait intervenir rien d'autre que le monde. Autant dire que nos mouvements doivent être caractérisés par cela que Patočka repérait comme simple 
condition de l'accueil, à savoir par l'impuissance. Le propre du vivant est qu'il est inachevé, qu'il n'est jamais pleinement lui-même, qu'il manque de lui-même ou est en défaut sur lui-même. C'est en ce sens qu'il faut entendre la remarque de Fink selon laquelle l'homme est la chose la plus finie. L'homme et, avec lui, les autres vivants est la chose la plus finie car il n'est pas fini. En d'autres termes, la néoténie biologique, que Patočka identifiait comme le ressort de l'acheminement vers la compréhension, doit être comprise comme l'expression d'une néoténie métaphysique, qui signifie l'incomplétude ontologique et donc l'inadaptation de tout vivant; de sorte que si l'homme est par excellence un animal néoténique, même s'il n'est pas le seul, c'est parce qu'il est le vivant par excellence loin de faire exception aux vivants. Le surgissement du vivant ne peut donc que renvoyer à une limitation qui affecte la surpuissance du monde mais dont elle ne peut être la source. Il relève de ce que j'ai nommé un archi-événement, événement d'une chute du monde lui-même, d'une déperdition de sa surpuissance. Rendre compte du vivant à partir du monde, c'est prendre acte d'un effondrement au cœur du monde, d'une privation qui excède le monde au sens où il ne peut en être la source mais qui, en tant qu'événement, ne relève pas d'une autre instance que lui-même. Le vivant procède d'une scission au cœur du monde et c'est pourquoi il lui appartient pleinement tout en en différant. Cet événement relève d'une métaphysique en un sens renouvelé et il faut donc compléter la cosmologie par la métaphysique au lieu de l'intégrer dans l'ontologie fondamentale.

\section{NOTES}

1. J. Patočka, Le monde naturel et le mouvement de l'existence humaine, trad. par E. Abrams, Dordrecht, Kluwer Academic Publishers, 1988, p. 102.

2. E. Fink, Spiel als Weltsymbol, trad. par Hildenbrand et Lindenberg (Le jeu comme symbole du monde), Paris, Minuit, 1966, p. 57.

3. J. Patočka, Le monde naturel et le mouvement de l'existence humaine, op. cit., p. 100. Cf. J. Patočka, Papiers phénoménologiques, trad. par E. Abrams, Grenoble, J. Millon, 1995, p. 114.

4. E. Fink, Le jeu comme symbole du monde, op. cit., p. 57.

5. J. Patočka, Le monde naturel et le mouvement de l'existence humaine, op. cit., p. 100.

6. J. Patočka, Papiers phénoménologiques, op.cit., p. 109.

7. Ibid., p. 101.

8. J. Patočka, Le monde naturel et le mouvement de l'existence humaine, op. cit. p. 267.

9. Ibid., p. 101.

10. J. Patočka, Papiers phénoménologiques, op.cit., p. 116.

11. J. Patočka, Le monde naturel et le mouvement de l'existence humaine, op. cit. p. 269. Cf. voir aussi J. Patočka, Papiers phénoménologiques, op.cit., p. 193 et J. Patočka, Platon et l'Europe, trad. par E. Abrams, Lagrasse, Verdier, 1983, p. 41-42.

12. J. Patočka, Le monde naturel et le mouvement de l'existence humaine, op. cit., p. 101.

13. J. Patočka, Le monde naturel et le mouvement de l'existence humaine, op. cit., p. 104.

14. Le premier mouvement correspond à la Befindlichkeit, le second à l'être-avec et le On et le troisième à la compréhension.

15. J. Patočka, Papiers phénoménologiques, op.cit. p. 109. 
16. Ibid., p. 110.

17. Ibid., p. 71. 